Gut and Liver, Vol. 10, No. 5, September 2016, pp. 661-662

EDITORIAL

\title{
Nonzero Risk of Hepatocellular Carcinoma Even after Sustained Virological Response
}

\author{
Hyun Woong Lee \\ Department of Internal Medicine, Chung-Ang University College of Medicine, Seoul, Korea
}

See "Prediction of the Risk of Hepatocellular Carcinoma in Chronic Hepatitis C Patients after Sustained Virological Response by Aspartate Aminotransferase to Platelet Ratio Index" by Keol Lee, et al. on page 796, Vol. 10. No. 5, 2016

\begin{abstract}
Approximately 130 to 150 million people are chronically infected with hepatitis $\mathrm{C}$ virus (HCV) in the world. HCV infection is a major cause of chronic hepatitis, liver cirrhosis and hepatocellular carcinoma (HCC). The existence of hepatitis Coriginally identifiable only as a type of non-A, non-B hepatitiswas suggested in the 1970s and proven in 1989. In the treatment of chronic hepatitis C (CHC), antiviral therapy has seen brilliant advances and rapid evolution in efficacy as well as safety and tolerability within recent several years. New antiviral agents now promise excellent sustained virological response (SVR) rates. ${ }^{1}$ After the widespread prescription of direct-acting antivirals (DAAs), the incidence of HCC will decrease gradually in the upcoming decades. However, the risk of HCC still remains in patients with advanced fibrosis, even though SVR reduces the progression to $\mathrm{HCC}^{2}$. Therefore, current guidelines recommended continued HCC surveillance for patients with liver cirrhosis even after SVR. ${ }^{3}$
\end{abstract}

In a recent meta-analysis of observational studies, SVR is associated with a reduction in the relative risk for HCC for persons at all stages of liver disease (hazard ratio, 0.24; $<<0.01$ ). ${ }^{4}$ This meta-analysis suggests that interferon (IFN) therapy is beneficial in reduction of HCC development after SVR. However, even if the absolute reduction in risk was $4.6 \%$, approximately $1.5 \%$ of the patients with SVR developed HCC. Up to now, there is no data supporting long-term benefits of SVR achieved with DAAs-based regimens in the risk of HCC development because of insufficient follow-up time. In the near future, DAA-based regimens will induce the high SVR rates and increase the numbers of patients with SVR. Consequently, a nonzero risk of HCC development after SVR may be the hot topic in many studies. ${ }^{5}$
Furthermore, it is very important to identify patients at high risk for developing HCC.

Several markers have been identified to predict the risk of HCC in CHC patients with SVR. For example, the pretreatment platelet count, alkaline phosphatase, and older age were significantly associated with the risk of HCC development. ${ }^{6}$ Another study reported that posttreatment alanine aminotransferase level $<40 \mathrm{U} / \mathrm{L}$ and $\alpha$-fetoprotein $<6.0 \mathrm{ng} / \mathrm{mL}$ were the important factors as high negative predictive values (NPV) for HCC development. $^{7}$ In recent study, diabetes (risk ratio, 2.08; $\mathrm{p}=0.0451$ ) and fibrosis 4 index at SVR 24 (risk ratio, 1.73; $p=0.0198$ ) were also predictive factors for HCC development even after SVR in noncirrhotic patients. ${ }^{8}$

In this issue of Hepatology, Lee et al. ${ }^{9}$ reported HCC development was observed among CHC patients who achieved SVR and the aspartate aminotransferase to platelet ratio index (APRI) could be a useful marker to classify HCC risk in CHC patients with SVR. As a result, pretreatment APRI and posttreatment platelet showed 100\% NPV at 5 years, and only pretreatment APRI showed 100\% NPV at 10 years. However, as authors mentioned, there are limitations to the data supporting the feasibility of pretreatment APRI related to prediction of HCC risk. This study is observational and retrospective study. Further limitation is the use of single-center study and the number of newly developed HCC patient was very small $(n=8)$. Nevertheless, these data over the past 8 years clearly demonstrated that the achievement of SVR definitely decreased the incidence of HCC development. Moreover, this study reminds physicians of the clinical usefulness of APRI, a simple, noninvasive marker for fibrosis, to predict patients needed regular HCC surveillance.

\footnotetext{
Correspondence to: Hyun Woong Lee

Department of Internal Medicine, Chung-Ang University College of Medicine, 102 Heukseok-ro, Dongjak-gu, Seoul 06973, Korea

Tel: +82-2-6299-1417, Fax: +82-2-6299-1137, E-mail: lhwdoc@hanmail.net

pISSN 1976-2283 eISSN 2005-1212 http://dx.doi.org/10.5009/gnl16340

@ This is an Open Access article distributed under the terms of the Creative Commons Attribution Non-Commercial License (http://creativecommons.org/licenses/by-nc/4.0) which permits unrestricted non-commercial use, distribution, and reproduction in any medium, provided the original work is properly cited.
} 
In view of the mechanism of IFN, it induces not only antiviral efficacy but also upregulation of antitumor immune responses, such as upregulation of natural killer cell activity and increasing the number of circulating T-helper lymphocytes. ${ }^{10}$ However, it remains an important question whether DAA-based regimens also have antitumor efficacy. In addition, it is not defined clearly whether oral antivirals can reduce the risk of HCC recurrence after curative resection or liver transplantation. It is also unclear whether the incidence of HCC development after SVR is different between DAA-based treatment and IFN-based treatment.

Our effort to select SVR patients at the highest risk of HCC can maximize the benefit of continued screening for SVR patients in view of cost-effectiveness. Therefore, multicenter, prospective trials with significant number of patients will be needed to clarify the durability of SVR after DAA-based treatment, the incidence and predictors of HCC development even after SVR.

Although this study may not be the new concept, this result provides the need for advanced studies with DAA-based therapy to stratify patients who can be intervened with more aggressive HCC screening and follow-up. Hopefully, such studies will improve the cost-effectiveness and detection of early HCC.

\section{CONFLICTS OF INTEREST}

No potential conflict of interest relevant to this article was reported.

\section{REFERENCES}

1. Pawlotsky JM. New hepatitis C therapies: the toolbox, strategies, and challenges. Gastroenterology 2014;146:1176-1192.
2. Yamashita N, Ohho A, Yamasaki A, Kurokawa M, Kotoh K, Kajiwara E. Hepatocarcinogenesis in chronic hepatitis C patients achieving a sustained virological response to interferon: significance of lifelong periodic cancer screening for improving outcomes. J Gastroenterol 2014;49:1504-1513.

3. AASLD/IDSA HCV Guidance Panel. Hepatitis C guidance: AASLDIDSA recommendations for testing, managing, and treating adults infected with hepatitis C virus. Hepatology 2015;62:932-954.

4. Morgan RL, Baack B, Smith BD, Yartel A, Pitasi M, Falck-Ytter Y. Eradication of hepatitis $\mathrm{C}$ virus infection and the development of hepatocellular carcinoma: a meta-analysis of observational studies. Ann Intern Med 2013;158(5 Pt 1):329-337.

5. Li DK, Chung RT. Impact of hepatitis C virus eradication on hepatocellular carcinogenesis. Cancer 2015;121:2874-2882.

6. Morgan TR, Ghany MG, Kim HY, et al. Outcome of sustained virological responders with histologically advanced chronic hepatitis C. Hepatology 2010;52:833-844.

7. Asahina Y, Tsuchiya K, Nishimura T, et al. Alpha-fetoprotein levels after interferon therapy and risk of hepatocarcinogenesis in chronic hepatitis C. Hepatology 2013;58:1253-1262.

8. Toyoda H, Kumada T, Tada T, et al. Risk factors of hepatocellular carcinoma development in non-cirrhotic patients with sustained virologic response for chronic hepatitis C virus infection. J Gastroenterol Hepatol 2015;30:1183-1189.

9. Lee K, Sinn DH, Gwak GY, et al. Prediction of the risk of hepatocellular carcinoma in chronic hepatitis C patients after sustained virological response by aspartate aminotransferase to platelet ratio index. Gut Liver 2016;10:796-802.

10. George PM, Badiger R, Alazawi W, Foster GR, Mitchell JA. Pharmacology and therapeutic potential of interferons. Pharmacol Ther 2012;135:44-53. 